\title{
A Survey of Power Aware Routing Schemes for Mobile Ad Hoc Networks
}

\author{
Muhammad Ibrahim \\ Channa \\ Quaid-e-Awam University of \\ Engineering, Science and \\ Technology, Pakistan
}

\author{
Akhtar Hussain \\ Jalbani \\ Quaid-e-Awam University of \\ Engineering, Science and \\ Technology, Pakistan
}

\author{
Pardeep Kumar \\ Harani \\ Quaid-e-Awam University of \\ Engineering, Science and \\ Technology, Pakistan
}

\begin{abstract}
The power aware routing schemes for mobile ad hoc networks have been developed for increasing network life time by isolating low power nodes from the routing process. This paper presents a survey of some recently proposed power aware routing schemes based on certain characteristics such as multipath support, quality of service support, mobility support and reliability support. It has been learnt that the majority of the proposed power aware routing schemes are not suitable for real-time applications, as they don't support the establishment of multiple simultaneous paths, provision of guaranteed quality of service, and random node mobility. Reliability has also been an important concern in those schemes, which needs to be incorporated in order to find trust aware energy efficient paths.
\end{abstract}

\section{General Terms}

Mobile ad hoc network, Power aware routing scheme.

\section{Keywords}

Mobile ad hoc network, power aware routing, residual energy, network lifetime, quality of service, multipath, mobility, reliability.

\section{INTRODUCTION}

Mobile ad hoc networks are autonomous systems, where each node operates as a host and as a router. It is multi-hop in nature, where, communication takes place between a given source and destination pair through intermediate relay nodes. There is no fixed infrastructure and the mobile hosts move randomly in various directions, resulting into an unpredictable network topology [1]. The mobile ad hoc networks are used in applications such as business meetings, crises management, search and rescue operations, army campaigns, academic activities and personal area networks.

The mobile nodes are battery operated and it is a challenging task to maximize their life time during critical applications such as emergency response. The traditional routing schemes for mobile ad hoc networks such as AODV [2], DSR [3] and DSDV [4] take into consideration the number of hops along a given route (hop count) as a route selection metric. A low power node on the shortest path may drain off its battery power and may disappear from the network during communications on the given path. The power aware routing schemes try to find a route, where each node along the given route has sufficient energy to accomplish the required communication task. The power aware routing schemes take into consideration the node's remaining battery power and other related cost metrics to find a route between a given source and destination pair [5]. The power aware routing metrics include minimum energy consumed per packet, maximum time to network partition and minimum variance in node power levels [6].

This paper presents a review of some existing power aware routing schemes for mobile ad hoc networks. The rest of the paper is organized as follows. Section 2 presents a brief description of various power aware routing schemes for mobile ad hoc networks. Section 3 presents a comparative analysis of various power aware routing schemes and section 4 concludes the paper.

\section{POWER AWARE ROUTING SCHEMES}

Several power aware routing schemes have been proposed for mobile ad hoc networks. In Power Aware On Demand Multipath Routing [5], an energy efficient shortest path is established between a given source and destination pair. The proposed scheme maintains the information about the nodes on a given route along with their corresponding residual energy levels. It maintains multiple routes simultaneously, so the switching from one route to another route does not create additional delay and packet loss. During route discovery, the duration of a path is estimated as the minimum residual energy of a link along the given route and is used as a cost function in path selection. During packet forwarding, the amount of conserved energy is updated by subtracting the energy consumed for forwarding a packet. If an active route breaks, an alternate route is selected for data transmissions.

The QoS Based Power Aware Routing Scheme [7] finds an energy efficient route, which meets QoS constraints along an established path. If the application doesn't require QoS guarantees, the protocol finds a path consisting of high energy nodes. If the QoS constrained path is required, the protocol considers node energy and link capacity as route selection metrics. During route discovery, the source node specifies the minimum energy and minimum bandwidth required along the established route. Each node inserts its residual energy and available bandwidth information into the route selection packets. The destination node finds and establishes the route which meets the energy and bandwidth constraints of the user application. If an active route breaks due to link failure or battery power depletion, the local route repair is used to find a new route.

The Power Aware Routing Algorithm [8] maximizes the network lifetime and minimizes the power consumption by establishing an energy efficient and congestion free route. The proposed scheme takes into consideration the accumulated 
energy of a path, status of battery life time and type of data to be transferred (real time and non-real time). During route discovery, the source node specifies the traffic type, total battery status and total traffic level in the routing packets. The traffic level of a node represents the number of packets buffered in the node's interface queue. Each node updates the relevant information in the routing packets and forwards them towards the destination node. The destination node buffers the routing packets for certain time interval and selects the route which meets the required power and link status ratio.

The Power Aware Routing Protocol [9] establishes an efficient, stable and long lasting path under large network size and high node mobility for a variety of data traffic. The power status of each node is periodically monitored and stored. The power level of a node is categorized as Active, Critical and Danger. The protocol comprises of Route Construction, Route Error and Recovery, and Route Eraser phases. During Route Construction, an stable path having all Active nodes is selected. If the link break occurs, the intermediate node performs local route repair to find an alternate Active route. Once the established Active route is undesired, the Route Eraser phase is initiated, where each node updates its routing table information by removing Active route entries.

The Power Aware QoS Multipath Routing Protocol [10] establishes a path, which meets the bandwidth and energy requirements of the application. If the application does not require QoS guarantees, the nodes with high energy level are selected along the established route. The proposed scheme comprises of Route Discovery, Route Selection and Route Maintenance phases. During Route Discovery, the application specifies the required bandwidth and required energy in the routing packets. If the intermediate node meets the bandwidth and energy requirements of the application, it forwards the packet to its next-hop and discards otherwise. The destination node replies back to the source node through maximum of 3 different routes. During Route Selection, the source node receives information regarding multiple routes to the destination and selects the best path by sorting multiple routes in descending order of node's residual energy and bandwidth. The route with maximal nodal residual energy is selected for data transfer. If the energy level of a node decreases than the required energy, the link is assumed to be broken and a new route setup is initiated.

The Variable Range Location Aided Routing [11] reduces the network energy consumption by using variable transmission power control. Each mobile node is equipped with a GPS device and the source node specifies the required energy in the route selection packets. The energy factor at each node is calculated, which is used to find an energy efficient path. The path energy factor is obtained by multiplying the energy factor of all nodes along a given path.

The Energy Aware Variable Transmission Range Routing [12] reduces the network energy consumption by varying the transmission range of nodes dynamically. During route discovery, each node specifies its current location in the routing packets generated by the destination node. Each intermediate node receives multiple routing packets from its downstream nodes, calculates the distance between itself and all its downstream nodes and selects the nearest node. The transmission energy is estimated based on the distance between current node and its nearest downstream node. This process continues until the route is established and data transmission takes place.
The Fuzzy Based Power Aware Routing [13] estimates the power consumed at a node for a particular data transmission through received signal strength. The battery cost function of each route is calculated and appended in the routing packets along with the node's power consumption. The destination node applies fuzzy logic and selects the route among all available routes having low power consumption. During mobility, if the nodes on an established route start moving away from each other, the probability of link break increases and an alternate route is established through route maintenance process.

The Location Based Power Aware Routing [14] finds an optimal path in terms of bandwidth and power consumption. The proposed scheme divides the network into Expected Zone and Request Zone. The Expected Zone is the region, where the destination node can move randomly. The Request Zone is the region, where the routing packets are broadcast to find a route to a given destination. During route discovery, if an intermediate node receives routing packet and it lies within the Request Zone, it rebroadcasts the packet and drops the packet otherwise. This process continues until the destination node receives routing packets and finds an energy and bandwidth efficient path.

The Residual Energy Based OLSR Protocol [15] selects an MPR node based on its residual energy and reachability. The proposed scheme aims at avoiding the selection of power deficient nodes as MPRs. During MPR selection, a node calculates the residual energy and reacheability of all its onehop neighbors, who are the candidates for MPRs. If a candidate MPR node possesses the highest residual energy among all its competitors and its reachability is also greater than zero, it is included in the MPR list.

\section{PERFORMANCE COMPARISON}

This section presents a comparative analysis of the proposed power aware routing schemes in terms of various characteristics such as multipath support, quality of service support, mobility support and reliability support as described in Table 1. Multipath support reduces the excessive route setup delay and packet loss. Quality of service support facilitates real-time applications. Mobility support helps in predicting the potential link break and taking appropriate action. Reliability support ensures that the nodes provide exact information about their residual energy during the route setup process.

The Power Aware On Demand Multipath Routing [5] and the Power Aware QoS Multipath Routing [10] support the establishment of multiple active routes. The excessive delay caused due to frequent route setup in case of link break and the packet loss may be decreased significantly. The QoS Based Power Aware Routing Scheme [7], Power Aware Routing Algorithm [8] and the Power Aware QoS Multipath Routing [10] schemes support real-time applications in terms of bandwidth requirements. The Power Aware Routing Protocol [9] and the Fuzzy Based Power Aware Routing [13] schemes take into consideration the node mobility, which helps in predicting the link break and taking an appropriate action. These schemes are feasible for the situations, where the node mobility is very frequent. None of the proposed schemes takes into consideration the node reliability. It is possible that during the route setup process, a selfish node may save its battery power/bandwidth by sending fake information about its residual energy/available bandwidth, and thus getting itself out of the packet forwarding process. 


\section{CONCLUSION}

This paper presents a review of some power aware routing schemes for mobile ad hoc networks. The proposed schemes have been analyzed in terms of certain characteristics such as multipath support, quality of service support, mobility support and reliability support. It has been observed that very few routing schemes support multipath, quality of service and node mobility, which are the key requirements of real-time applications. All the proposed schemes are lacking the provision of node reliability, which helps in identifying selfish nodes and taking an appropriate action against them. It is worthwhile to extend the proposed schemes in such a way that the node reliability is also considered along with other characteristics during the route setup process.

\section{REFERENCES}

[1] Forman, G. and Zahorjan, J., "The Challenges of Mobile Computing". IEEE Computer, 1994, 27(4), 38-47.

[2] Perkins, C.E. and Royer, E.M. 1999. Ad Hoc On Demand Distance Vector Routing. In Proceedings of 2nd IEEE Workshop on Mobile Computing Systems and Applications, New Orleans, LA, 90-100.

[3] Johnson, D.B. and Maltz, D.A., "Dynamic Source Routing in Ad-Hoc Wireless Networks". Mobile Computing, Kluwer Academic Publishers, 1996, Chapter $5,153-181$.

[4] Perkins, C.E. and Bhagwat, P. 1994. Highly Dynamic Destination-Sequenced Distance-Vector Routing (DSDV) for Mobile Computers. In Proceedings of ACM SIGCOMM, London, U.K., 234-244.

[5] Rajaram, A. and Sugesh, J. "Power Aware Routing for MANET using On-Demand Multi-Path Routing Protocol". International Journal of Computer Science Issues, 8(4), 2011, 517-522.

[6] Singh, S., Woo, M. and Raghavendra, C.S. 1998. PowerAware Routing in Mobile Ad Hoc Networks. In Proceedings of the International Conference on Mobile Computing and Networking, Dallas, Texas, 181-190.
[7] Rishiwal, V., Verma, S. and Bajpai, S.K., "QoS Based Power Aware Routing in MANETs". International Journal of Computer Theory and Engineering. 1(1), 2009, 47-54.

[8] Rishiwal, V., Yadav, M., Verma, S. and Bajapai, S.K., "Power Aware Routing in Ad Hoc Wireless Networks". Journal of Computer Science and Technology, 9(2), 2009, 101-109.

[9] Ashwani, K., Gupta, P. and Chauhan, R., "Power Aware Routing in Ad Hoc Networks". Transactions on Internet Research, 1(2), 2005, 70-75.

[10] Santhi, S. and Sudha, S.G., "Power Aware QoS Multipath Routing Protocol for Disaster Recovery Networks". International Journal of Wireless \& Mobile Networks (IJWMN), 3(6), 2011, 47-57.

[11] Joshi, N.N. and Joshi, R.D., "Energy Conservation in Manet using Variable Range Location Aided Routing Protocol". International Journal of Wireless \& Mobile Networks (IJWMN), 3(5), 2011, 265-280.

[12] Nayak, P., Agrawal, R. and Verma, S., "Energy Aware Routing Scheme for Mobile Ad Hoc Network using Variable Range Transmission". International Journal of Ad hoc, Sensor \& Ubiquitous Computing (IJASUC), 3(4), 2012, 53-63.

[13] Abirami, S., Bhanumathi, V. and Dhanasekaran, R 2012. A Balanced Approach for Power Aware Routing in MANET using Fuzzy Logic. In Proceedings of International Conference on Recent Trends in Computational Methods, Communication and Controls.

[14] Suri, P.K., Soni, M.K. and Tomar, P., "Framework for Location Based Power Aware Routing in MANET". International Journal of Computer Science Issues, 8(3), 2011, 461-466.

[15] Hirata, W.K., Higami, Y. and Kobayashi, S., "REOLSR: Residual Energy-Based OLSR Protocol in Mobile Ad Hoc Networks". International Journal of Multimedia Technology, 1(2), 2011, 93-97.

Table 1: Comparative Analysis of Power Aware Routing Schemes

\begin{tabular}{|c|c|c|c|c|}
\hline Routing Scheme & $\begin{array}{c}\text { Multipath } \\
\text { support }\end{array}$ & $\begin{array}{c}\text { QoS } \\
\text { support }\end{array}$ & $\begin{array}{c}\text { Mobility } \\
\text { support }\end{array}$ & $\begin{array}{c}\text { Reliability } \\
\text { support }\end{array}$ \\
\hline Power Aware On Demand Multipath Routing [5] & Yes & No & No & No \\
\hline QoS Based Power Aware Routing [7] & No & Yes & No & No \\
\hline Power Aware Routing Algorithm [8] & No & Yes & No & No \\
\hline Power Aware Routing Protocol [9] & No & No & Yes & No \\
\hline $\begin{array}{c}\text { Power Aware QoS Multipath Routing Protocol } \\
{[10]}\end{array}$ & Yes & Yes & No & No \\
\hline Variable Range Location Aided Routing [11] & No & No & No & No \\
\hline Energy Aware Variable Transmission Range & No & No & No & No \\
\hline Routing [12] & No & No & Yes & No \\
\hline Fuzzy Based Power Aware Routing [13] & No & No & No & No \\
\hline Residual Energy Based OLSR Protocol [15] & No & No & No & Non Based Power Aware Routing [14] \\
\hline
\end{tabular}

